\title{
A novel platform for heterologous gene expression in Trichoderma reesei (Teleomorph Hypocrea jecorina)
}

\author{
Mikael Skaanning Jørgensen ${ }^{1}$, Dominique Aubert Skovlund ${ }^{2}$, Pia Francke Johannesen ${ }^{2}$ and Uffe H Mortensen ${ }^{1 *}$
}

\begin{abstract}
Background: The industrially applied filamentous fungus Trichoderma reesei has received substantial interest due to its highly efficient synthesis apparatus of cellulytic enzymes. However, the production of heterologous enzymes in $T$. reesei still remains low mainly due to lack of tools for genetic engineering.

Results: In this study we present new genetic tools for T. reesei to further expand its use in industrial production. We have developed an expression platform where genes are inserted into a versatile expression vector via highly efficient uracil-excision cloning and subsequently inserted into a defined position in the $T$. reesei genome ensuring that enzyme production from different transformants can be directly compared. The ade2 locus was selected as integration site since ade2 mutants develop red pigment that facilitates easy and rapid detection of correctly targeted transformants. In addition, our system includes a tku70 disruption to increase gene targeting efficiency and a new bidirectional marker, pyr2, for iterative gene targeting. The dual selection system, color and prototrophism, ensures that correct transformants containing the desired gene inserted into the defined expression site can be selected with an efficiency approaching 100\%.
\end{abstract}

Conclusions: The new genetic tools we have developed are suitable for high-throughput integration of genes into the genome of $T$. reesei and can easily be combined with techniques for generation of defined mutants. Moreover, the usability of the novel expression system with ade2 as integration site was confirmed by expression of a Thermomyces lanuginosus lipase.

Keywords: Expression, pyr2, Trichoderma reesei, Defined integration

\section{Background}

Trichoderma reesei is a key workhorse for commercial scale production of different enzymes used by the bioethanol, pulp, paper, and textile processing industries. This status is a consequence of its unique capability of producing and secreting large amount of enzymes, its amenability to large-scale fermentation as well as its long history of safe use in industrial enzyme production. Importantly, production of several $T$. reesei enzymes has obtained the generally recognized as safe (GRAS) status by the U.S. Food and Drug Administration (FDA). Moreover, T. reesei serves as a model organism for the regulation of expression and biochemistry of (hemi-)

\footnotetext{
* Correspondence: um@bio.dtu.dk

${ }^{1}$ Department of Systems Biology, Center for Microbial Biotechnology,

Technical University of Denmark, Building 223, DK-2800 Lyngby, Denmark

Full list of author information is available at the end of the article
}

cellulose degradation enzymes and pathways $[1,2]$. There is an increasing demand for these enzymes since they are employed for the saccharification of cellulosic plant biomass to simple sugars for biofuel production. As enzymes constitute an important cost in the production of bioethanol, large research efforts as well as large government funding have aimed to continuously improve $T$. reesei as an enzyme production host. This process will be greatly facilitated by the fact that its genome has recently been sequenced setting the stage for strain development by directed genetic engineering [3].

Unfortunately, the highly efficient protein synthesis machineries of $T$. reesei, which enable yields of homologous proteins in excess of $100 \mathrm{~g} / \mathrm{l}$ [4], has so far not prevailed for synthesis of heterologous proteins and yields remain low [5]. Methods and tools to improve synthesis of heterologous proteins in this fungus are therefore highly desirable. 
One bottle neck towards this goal has been the low efficiency of gene targeting in T. reesei, but like in a number of other fungi this problem has been dramatically reduced by deleting a gene involved in Non-Homologous End-Joining (NHEJ). In such strains the frequency of successful integration by gene targeting was first reported to increase to $>95 \%$ from the $5-10 \%$ obtained with wildtype strains [6]. However, there is still room for improvement as a recent study in $T$. reesei has demonstrated that the efficiency of homologous integration in NHEJ deficient strains can be highly site specific and may vary from 33 to $100 \%$ depending on insertion site [7].

Before T. reesei can be routinely used for heterologous protein production, it is necessary to gain insights into e.g. the influence of promoter sequences, effects of codon optimized synthetic genes, or expression rates of selected orthologous genes. This type of analysis requires construction of large numbers of strains where the genes to be compared are expressed from a defined locus in isogenic strains to allow for proper comparisons. Moreover, such analysis may often include experiments that require multiple rounds of genetic engineering in the same strain. Consequently, the need for molecular tools that allow easy genetic modifications of $T$. reesei for industrial strain development are urgent. We have therefore developed a new expression platform in $T$. reesei that facilitates heterologous gene expression from a defined locus with an improved gene-targeting throughput. Our expression platform is composed of four parts: 1) a versatile integration plasmid for gene expression containing a USER (Uracil-Specific Excision Reagent) cassette for highly efficient ligase-free USER cloning, 2) a bidirectional marker pyr2, encoding orotate phosphoribosyl transferase, that allows for iterative gene targeting, 3) a $t k u 70$ gene disruption strain for efficient gene targeting, and 4) a color marker that facilitates identification of correctly targeted strains, even in early stages of colony development. The use of pyr 2 as a bidirectional marker has not previously been reported for $T$. reesei, but we here demonstrate that it serves as a highly reliable alternative to the previously used pyr4 gene [8], encoding orotidine-5' -monophosphate decarboxylase. The applicability of the expression system was confirmed by expression of a Thermomyces lanuginosus lipase as reporter protein.

\section{Results and discussion}

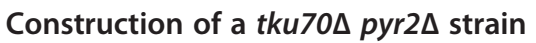

First, the $t k u 70$ gene involved in NHEJ was disrupted in the original T. reesei QM6a isolate by transforming this strain with a gene-targeting substrate based on the amdS marker; see Materials and methods for details. Sixty transformants were obtained and a subsequent PCR analysis (data not shown) identified a single transformant containing the desired disruption of tku70. The correct integration of amdS into tku70 in this transformant was confirmed by Southern blotting (Additional file 1: Figure S1) and the resulting strain was named MJ-T-001.

\section{pyr2 as a bidirectional marker}

Orthologous genes of the pyr2 gene have been used as bidirectional markers in several species such as Candida guillermondii, Schwanniomyces alluvius and Haloferax volcanii [9-11] based on the principle that 5-Fluoroorotic acid (5-FOA) is metabolized to 5-Fluorodeoxyuridine monophosphate (5-FdUMP), a suicide inhibitor of thymidylate synthase essential for DNA synthesis, in a process that depends on the orotate phosphoribosyl transferase activity of PYR2 [12,13]. To investigate the possibility that pyr 2 could also be used as a bidirectional marker in $T$. reesei, we deleted the entire coding sequence (CDS) of pyr2 (DDBJ/EMBL/GenBank protein accession number: EGR51642.1, JGI ID: 21435) in MJ-T-001. In this case, the gene-targeting substrate did not contain a selectable marker as we hypothesized that correctly targeted strains, unlike wild-type strains, would survive exposure to 5FOA. Eleven transformants were obtained after transformation with the pyr2 deletion construct on 5-FOA plates, but only three of these displayed normal growth rates. As expected for correctly targeted strains these three transformants were all uridine auxotroph as they required addition of uridine to the media in order to grow [14-16]. Finally, deletion of pyr2 was confirmed in one of the three transformants by PCR (data not shown) and by Southern blotting (Figure 1 and Additional file 2: Figure S2). The resulting strain was named MJ-T-006.

Two gene-targeting substrates were constructed to determine whether pyr 2 could serve as bidirectional marker. One was designed to eliminate pks4 (DDBJ/ EMBL/GenBank protein accession number: EGR44538.1, JGI ID: 82208), a gene that encodes the polyketide synthase necessary for production of green conidial pigment [17]; and ade2 (DDBJ/EMBL/GenBank protein accession number: EGR49709.1, JGI ID: 105832), an ortholog of the ade2 gene in Saccharomyces cerevisiae, which encodes a phosphoribosylaminoimidazole carboxylase necessary for production of purines. Lack of this enzyme in species such as S. cerevisiae, Pichia pastoris and Aspergillus oryzae [18-20] results not only in adenine auxotrophy, but also in development of red colonies as the precursor 5aminoimidazole ribonucleotide accumulates in the cell and polymerizes [21]. Both gene targeting substrates were based on a pyr2 selectable marker, which was flanked by $375 \mathrm{bp}$ direct repeats, originating from the $A$. oryzae pyr4 promotor region, to allow for subsequent marker excision by direct repeat recombination, see Figure 2 .

Transformation of the $p k s 4$ gene-targeting substrate into MJ-T-006 produced 29 uracil prototroph transformants on Trichoderma minimal medium (MM) plates. After the 


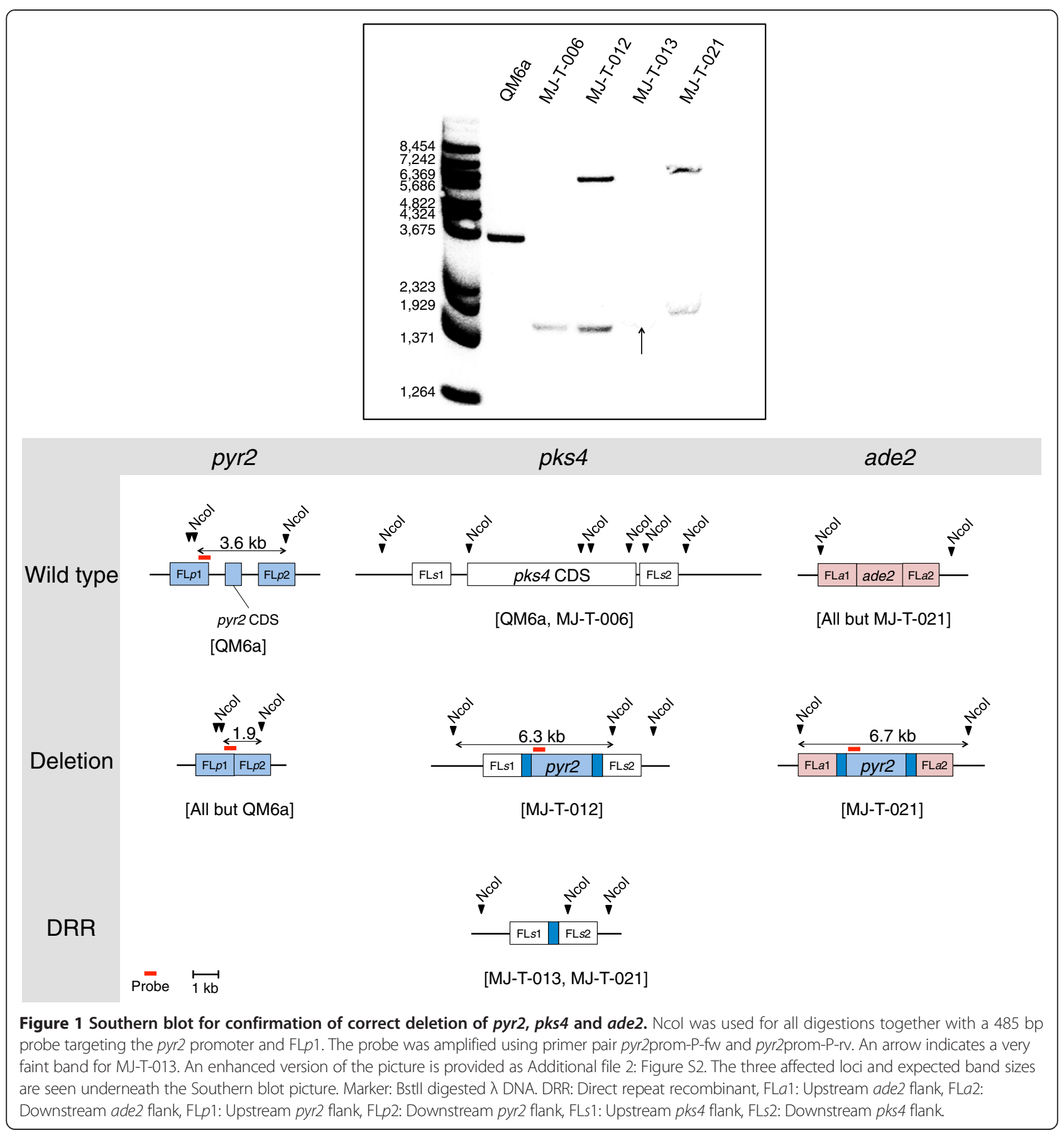

transformants were transferred to PDA plates for sporulation, we observed that eleven $(\sim 40 \%)$ of the transformants lacked pigment production and were white as expected for $p k s 4$ deleted strains (Figure 3). This strongly indicates that pyr 2 is an efficient marker for positive selection. In agreement with this view, PCR analysis of the white transformants confirmed that $p k s 4$ was indeed deleted in all cases. Moreover, one of the white transformants, named MJ-T-012, was also confirmed by Southern blotting
(Figure 1). The core of the gene targeting substrate contains pyr 2 flanked by direct repeats and the pyr2 marker can therefore be eliminated by direct repeat recombination. To demonstrate that the pyr 2 gene can also be counter-selected, approximately $10^{8}$ spores from this strain were inoculated on plates supplemented with 5-FOA and uridine. Four colonies were isolated after five days of incubation and subsequent PCR- and sequencing analyses demonstrated that the pyr 2 gene was lost in all 

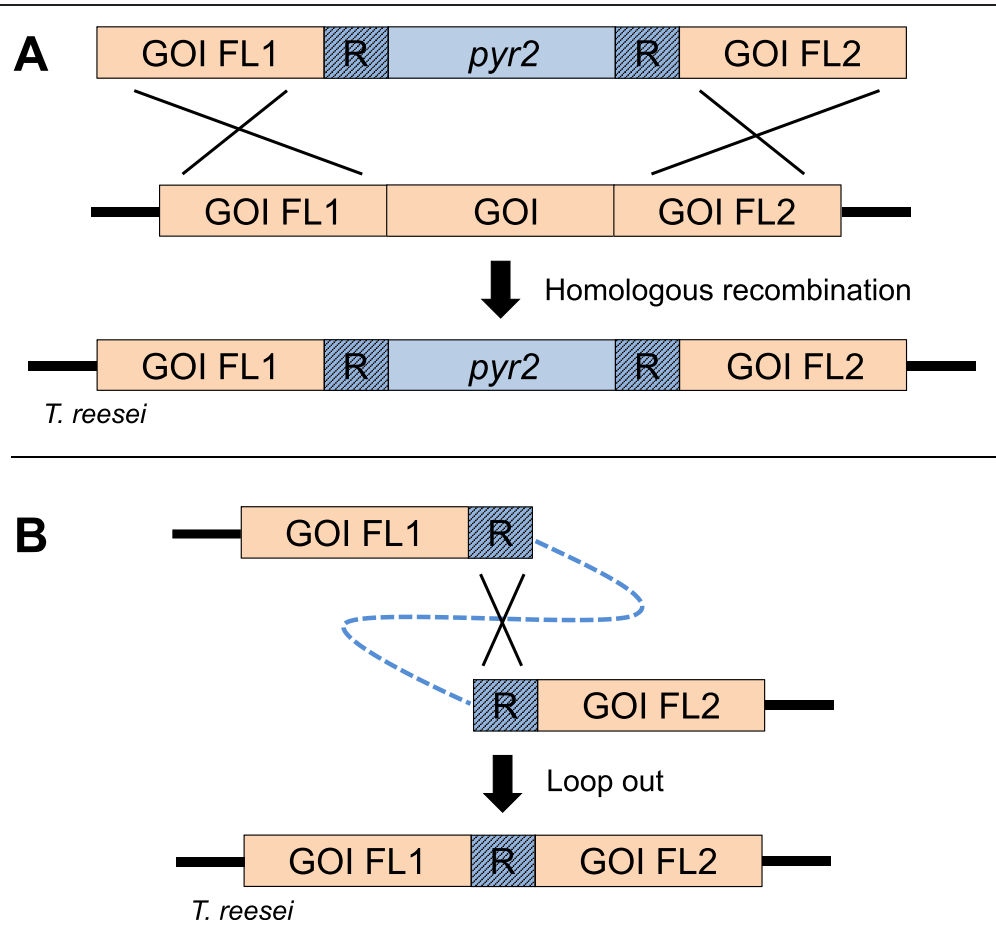

Figure 2 Use of pyr2 for genetic deletion and direct repeat recombination. A: Genetic deletion using pyr2 as selectable marker. B: Elimination of pyr2 by direct repeat recombination, selected for by subjection to 5-FOA. GOI: Gene of interest, FL1: Upstream flank, FL2: Downstream flank, R: Direct repeats.

four strains as the result of recombination between the direct repeats. In agreement with these results, the four strains were also displaying a uridine auxotrophic phenotype. One of these colonies was named MJ-T-013 and used for further experiments.

To demonstrate that pyr 2 can be used as a marker for iterative gene targeting, we transformed MJ-T-013 with a gene targeting substrate designed to delete ade2. Nine uracil prototrophic transformants appeared on the primary transformation plates after transformation. Of these nine transformants, four developed orange/red coloration after four days of growth, indicating successful deletion of the ade 2 gene (Figure 3). All nine colonies were tested for adenine auxotrophy and as expected, the four red colonies were all adenine auxotrophs whereas the remaining five were not. Correct deletion of ade 2 in the four selected red transformants was confirmed by PCR (data not shown). One of the four transformants containing the $p k s 4$ and ade 2 double deletion was verified by Southern blotting (Figure 1) and named MJ-T-021. Successful construction of $p k s 4$ and ade 2 double deletion strains demonstrate that pyr 2 can be used as an alternative to pyr4 for iterative gene targeting. Importantly, the coding sequence of pyr2 is $435 \mathrm{bp}$ shorter than that of pyr4, and as a consequence episomal vectors and gene targeting substrates that are based on pyr2 will be smaller than if they are based on pyr4, which is often advantageous.
A versatile integration vector for gene expression at ade2 In agreement with other studies, e.g. [6,7,14-16,22], we find that the gene targeting efficiency is quite high in strains with defective NHEJ. For both $p k s 4$ and ade2, we obtained deletions with an efficiency of $\sim 40 \%$. Although this success rate is sufficient for small-scale experiments, it may hamper using $T$. reesei as a high-throughput screening platform for the discovery and production of novel enzymes. However, the fact that ade 2 deletion strains rapidly develop red color as they grow on MM plates may simplify screening. Specifically, we envisioned that by inserting genes for heterologous expression into ade2, correctly targeted transformants can be easily selected as red colonies. Importantly, we find that deletion of ade 2 does not have a negative impact on the growth rate on MM medium supplemented with adenine as judged by race tube experiments (Table 1); and the locus is therefore well-suited for gene insertion and expression. We therefore decided to exploit the red/white color-screen offered by the ade 2 locus and incorporate it as a central part of our expression platform. Consequently, we constructed a versatile integration vector, pMJ-023, see Figure 4A, which in a single cloning step equips a gene of interest (GOI) with all necessary parts for integration and expression from ade2. Hence, the vector contains gene-targeting sequences for integration into ade 2 and the pyr 2 marker for selection/counter selection. Moreover, it contains a 


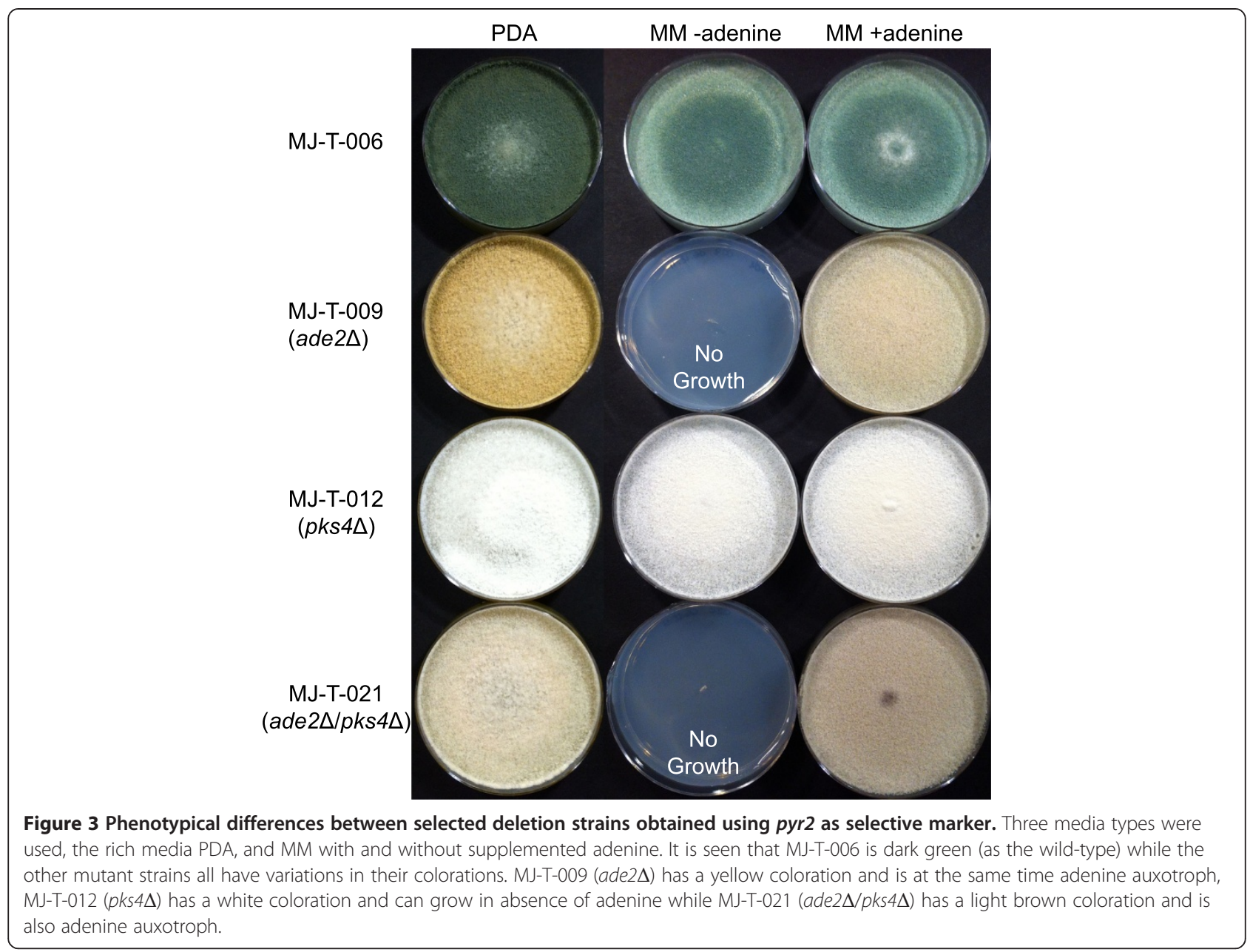

USER cloning cassette [23,24] flanked by the Aspergillus nidulans PgpdA promoter and a TtrpC terminator to allow for gene expression. Insertion of a GOI into the USER cassette by USER cloning or USER fusion, see Figure 4B, not only facilitates highly efficient vector construction in a manner compatible with high-throughput experiments, but also offers a simple routine for introducing additional genetic manipulations like site-directed mutagenesis, or addition of tagging sequences encoding epitope-, GFP-, or purification tags. A simple restriction enzyme digest of the resulting vector liberates the genetargeting substrate for integration into ade 2 by homologous recombination, see Figure 4C.
To demonstrate this concept we decided to express the T. lanuginosus lipase gene, lip, from the ade2 locus. Accordingly, lip was positioned between the A. nidulans PgpdA promoter and TtrpC terminator in pMJ-023, resulting in $\mathrm{pMJ}-051$. The gene-targeting substrate containing the PgpdA::lip::TtrpC fragment was liberated from the vector backbone by restriction enzyme digestion and inserted into ade 2 using pyr2 as selection marker. After transformation, several red colonies appeared and correct insertion of the expression construct in three of these transformants was confirmed by PCR and Southern blot analyses (Additional file 3: Figure S3). The three transformants, MJ-T-033-1, -2 and -3 , were analyzed for

Table 1 Growth rates of mutant strains in MM supplemented with $0.5 \mathrm{mM}$ adenine

\begin{tabular}{|c|c|c|c|}
\hline Strain & MJ-T-001 & MJ-T-009 & MJ-T-021 \\
\hline Genotype & pyr2 & 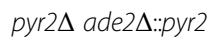 & pyr2 $\Delta$ pks $4 \Delta$ ade $2 \Delta:$ pyr2 \\
\hline Growth rate $(\mathrm{mm} / \mathrm{h})$ & 0.91 (st.dev. 0.04) & 0.99 (st.dev. 0.02) & 0.91 (st.dev. 0.09) \\
\hline
\end{tabular}

All strains are tku70::amdS. Genetic differences in genotype are as indicated. 

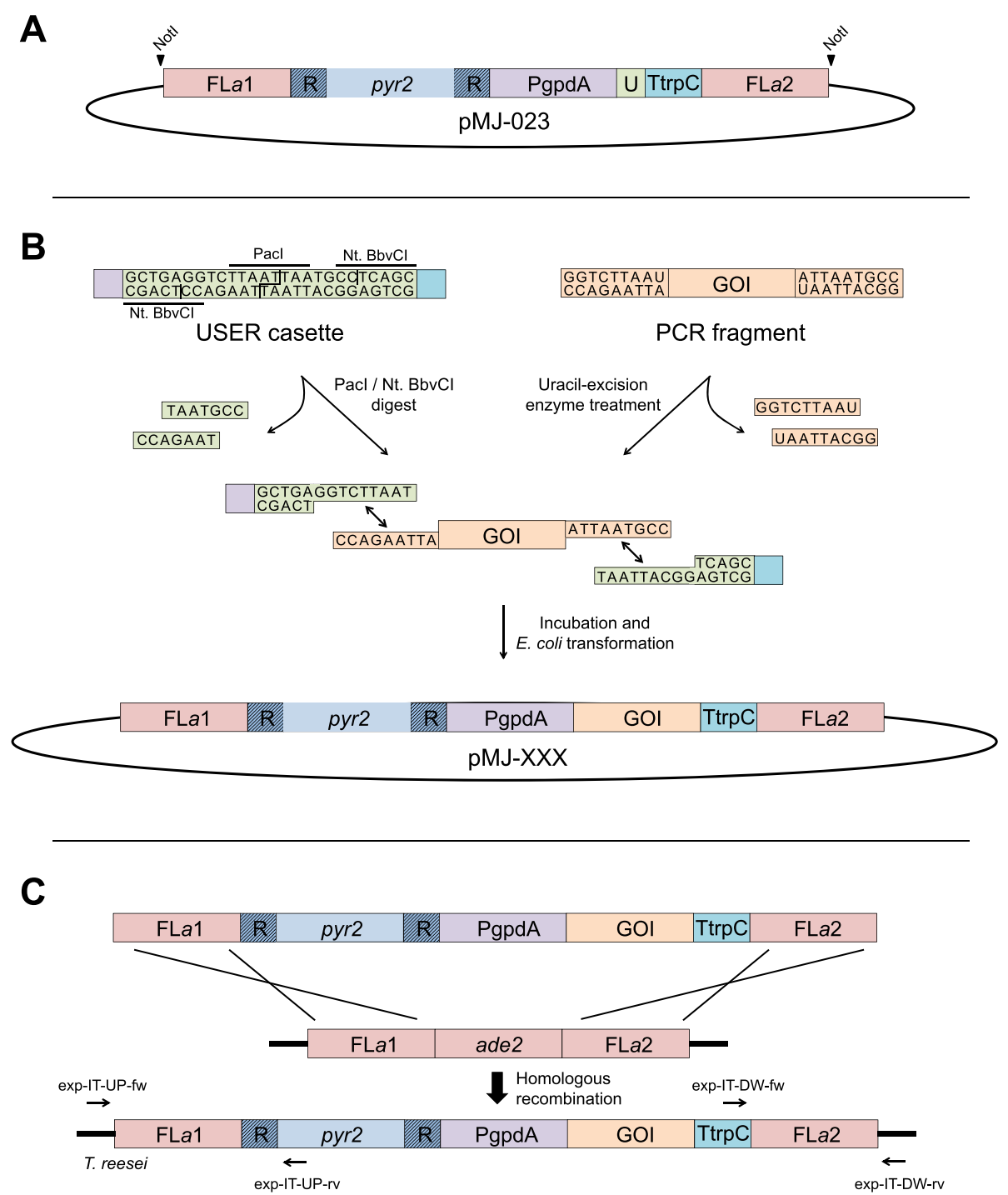

Figure 4 A versatile integration vector to facilitate gene expression in the ade2 locus. A) The pMJ-023 vector contains gene-targeting sequences (FLa1: upstream region of ade2 and FLa2: downstream region of ade2) for integration into ade2 locus (in pink); a pyr2 selectable/counter selectable marker (in light blue) flanked by direct repeats (in striped blue); and a USER cassette (in green) flanked by the PgpdA promoter (in purple) and the TrpC terminator (in turquois). The Notl cut sites that are used for excising the gene-targeting substrate are indicated. The E. coli vector backbone is marked in black; see Materials and methods for more information. B) The gene of interest (GOI) (orange) is inserted into the vector by USER cloning. C) The liberated gene-targeting substrate containing the GOI expression cassette integrates into ade2 by homologous recombination, in a process that results in ade2 deletion. Primer targets for diagnostic PCRs are indicated.

T. lanuginosus lipase production using a well-developed assay for measuring lipase activity, see Materials and methods. In a parallel experiment, an empty expression construct containing PgpdA::TtrpC was inserted into ade2 to generate strain MJ-T-020, which was analyzed in the same manner. The average lipase activities (Lipase Units, LU) for MJ-T-020, MJ-T-033-1, -2 and -3 were $1.19 \mathrm{LU} \pm$ $0.05,2.00 \mathrm{LU} \pm 0.15,1.85 \mathrm{LU} \pm 0.09$ and $2.09 \mathrm{LU} \pm 0.15 \mathrm{pr}$. $10 \mu \mathrm{l}$ supernatant, respectively. Hence, a significant and reproducible increase in the amounts of lipase activity could be detected in the strains expressing the PgpdA controlled lip. Specifically, additional lipase activity corresponding to an average of $0.79 \mathrm{LU}$ was produced in these strains.

\section{Conclusions}

In summary, we have developed an expression platform, which is designed for high-throughput construction of defined integrated $T$. reesei strains suitable for setup of large expression studies. The compatibility of USER cloning with PCR enables easy site-directed mutagenesis, promoter swaps, and epitope- and GFP tagging for protein engineering, characterization, purification, and 
Table 2 Overview of the strains used in this study

\begin{tabular}{|c|c|c|c|c|c|}
\hline \multirow[t]{2}{*}{ Strain } & \multirow[t]{2}{*}{ Genotype } & \multirow{2}{*}{$\begin{array}{l}\text { Growth in } \\
\text { presence of 5-FOA }\end{array}$} & \multicolumn{2}{|c|}{ Prototrophy } & \multirow[t]{2}{*}{ Reference } \\
\hline & & & Uridine & Adenine & \\
\hline QM6a & WT & - & + & + & (Martinez et al., 2008) [3] \\
\hline MJ-T-001 & tku70 ::amds ${ }^{+}$ & - & + & + & This study \\
\hline MJ-T-006 & tku70":amds ${ }^{+}$pyr $2 \Delta$ & + & - & + & This study \\
\hline MJ-T-009 & tku70::amdS ${ }^{+}$pyr2 $2 \Delta$ ade2 $\Delta:$ :pyr2 & - & + & - & This study \\
\hline MJ-T-012 & 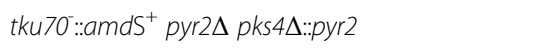 & - & + & + & This study \\
\hline MJ-T-013 & tku70::amdS ${ }^{+}$pyr $2 \Delta p k s 4 \Delta$ & + & - & + & This study \\
\hline MJ-T-020 & tku70-::amdS ${ }^{+}$pyr2 $\Delta$ ade2 $\Delta:: P g p d A-T t r p C-p y r 2$ & - & + & - & This study \\
\hline MJ-T-021 & 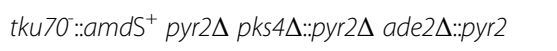 & - & + & - & This study \\
\hline MJ-T-033 & tku70":amdS ${ }^{+}$pyr2 $\Delta$ ade2 $\Delta \because:$ PgpdA-lip-TtrpC-pyr2 & - & + & - & This study \\
\hline
\end{tabular}

5-FOA: 5-Fluoorotic acid.

The genotypes are listed together with selected phenotypic characteristics.

production. Moreover, selected mutants can be further analyzed/optimized by iterative gene targeting using pyr2 as a bidirectional selective marker.

\section{Materials and methods Strains and media}

The strains included in this study are listed in Table 2 . Yeast extract-peptone-dextrose and Trichoderma Minimal Media (MM) was made as in Fink \& Hicks [25] and Gruber et al., 1990 [26], respectively. For composition of MM including additional supplements, see Additional file 4: Table S1. Potato dextrose agar (PDA) was obtained from BD/Difco.

\section{Gene identification}

The putative $T$. reesei proteins encoded by ade2 (JGI ID: 105832), pyr2 (JGI ID: 21435) and pks4 (JGI ID: 82208) were identified by BLASTing i) the 250 amino acid residues (aa) of the A. oryzae PyrF protein (/EMBL/GenBank protein accession number XM_001821908.2), ii) the 2157 aa sequence of the $A$. nidulans Ywa1 protein and iii) the 571 aa sequence of the $S$. cerevisiae Ade2 protein, respectively, in the JGI $T$. reesei filtered model proteins database (http://genome.jgi-psf.org/pages/blast.jsf?db=Trire2). The corresponding gene sequences were subsequently extracted from the same source.

\section{Vector construction}

An overview of the primers used for vector construction and the resulting plasmids are listed in Additional file 5: Table S2 and Additional file 6: Table S3, respectively. PCR fragments used for the constructions were made by using PfuX7 polymerase [27] and purified from agarose gel using the GFX kit (GE healthcare, Little Chalfont, United Kingdom). Specifically, pMJ-001 was made by inserting the relevant PCR fragment (see Additional file 7: Figure S4 and Additional file 5: Table S2) into pCR2.1 (Invitrogen) by topoisomerase-mediated ligation according to the instructions provided by the manufacturer. pMJ-005 was made by inserting the relevant PCR fragment into a StuI and SnaBI vector fragment of pMJ001 using In-Fusion ligation (Clontech). The remaining vectors were made by fusing PCR fragments with the pU1111-1 vector backbone [23] by restriction enzyme and ligase independent uracil-excision cloning [24] using the USER $^{\mathrm{Tu}}$ Friendly Cloning Kit protocol (New England Biolabs), see Additional file 5: Table S2 and Additional file 6: Table S3 for details. All vectors were cloned by transformation into chemically competent Fusion-Blue E. coli cells (Clontech, Mountain View, USA) as described in the In-Fusion ${ }^{\bullet}$ Dry-Down PCR Cloning Kit Protocol-at-a-Glance.

\section{Strain construction}

T. reesei protoplastations and transformations were performed as described by Gruber and co-workers [26]. Approximately $10 \mu \mathrm{gg}$ linearized DNA by restriction endonucleases was used for each transformation. Correct targeted integration was verified by diagnostic PCRs and Southern blots; see primers in Additional file 8: Table S4. All strains were incubated on solid media for five days at $28^{\circ} \mathrm{C}$; and in liquid media for 48 hours at $30^{\circ} \mathrm{C}$ and 200 RPM. The exception was for lipase assays as described later.

The gene targeting substrate for construction of MJ-T001 was obtained by digesting pMJ-005 with ClaI. This fragment was transformed into QM6a and transformants selected by using the amdS selection marker on MM plates supplemented with acetamide. All remaining genetargeting substrates were liberated from the plasmid backbone by NotI digestion and gel purified. MJ-T-006 was generated by transforming MJ-T-001 with the gene targeting substrate obtained from pMJ-017. In this case transformants were selected by plating on MM plates containing 5-FOA and uridine. MJ-T-009 and MJ-T- 
012 was obtained by transforming MJ-T-006 with gene targeting substrates isolated from pMJ-031 and pMJ-030, respectively. MJ-T-009 was selected on MM supplemented with adenine and MJ-T-012 on MM. MJ-T-013 was generated from MJ-T-012 by selecting for recombinants where the pyr 2 marker has been lost by direct repeat recombination. Specifically recombinants were selected by plating $10^{8}$ spores on 5 -FOA MM plates.

The pyr2 loop out strain, MJ-T-013, was transformed with the gene targeting substrate isolated from pMJ-031 and transformants selected on MM plates to acquire MJ-T-021. MJ-T-020 was obtained by transforming MJT-006 with the gene targeting substrate isolated from pMJ-023 and plating on MM plates. After transforming MJ-T-006 with the gene targeting substrate originating from pMJ-051, MJ-T-033-1, -2 and -3 were isolated from MM plates.

All transformants were streak-purified on the proper selection medium before further analysis. All strains were verified by diagnostic PCR and Southern blot, Figure 1, Additional file 1: Figure S1, Additional file 3: Figure S3 and Additional file 8: Table S4.

\section{Growth rate measurements}

The linear growth rates of MJ-T-001, MJ-T-009, MJ-T-012 and MJ-T-021 were measured in race tubes, as described by White and Woodward [28].

\section{Expression of T. lanuginosus lipase from the ade2 site}

The T. lanuginosus lipase gene (lip) (accession no. AF0 54513) was kindly provided by Jan Lehmbeck, Novozymes, Bagsvaerd. The lipase was produced by growing MJ-T033-1, -2 and -3 in $10 \mathrm{ml} \mathrm{YPD}$ at $30^{\circ} \mathrm{C}$ for four days at $200 \mathrm{rpm}$. At this point, the amount of lipase in the medium was determined by measuring the esterase activity. Specifically, the rate of p-nitrophenol formation using $p$-nitrophenyl valerat as substrate was measured in microtiter plate wells by mixing $10 \mu \mathrm{l}$ supernatant, $20 \mu$ dilution buffer (50 mM Tris/HCL ( $\mathrm{pH} 7.5), 10 \mathrm{mM}$ $\mathrm{CaCl}_{2}$ and $0.075 \%$ Brij-35 (Thermo Fisher Scientific, Rockford, USA)) and $200 \mu \mathrm{l}$ substrate solution (0.6 mM 4Nitrophenyl valerate (Sigma N4377) dissolved in methanol).

The Lipase Units (LU) were measured in an ELISA reader as absorption at $405 \mathrm{~nm}$ (peak absorbance of p-nitrophenol) in 30-second intervals for 40 minutes. The amount of LU in the samples was calculated based on included standards.

\section{Additional files}

Additional file 1: Figure S1. Southern blot for confirmation of tku70 truncation. Xbal was used for all digestions. The 900 bp probe targeting the tku70 CDS was amplified using primers tku70-P-fw and tku70-P-rv. Marker: Bstll digested lambda DNA.

Additional file 2: Figure S2. Enhanced version of Figure 1. An arrow indicates the band for MJ-T-013 that was not visible in Figure 1.

Additional file 3: Figure S3. Southern blots for confirmation of correct insertion of the lip expression construct. BamHI and Nhel were used for all digestions. A: Southern blot using two probes. One probe targeting FLa1 and the other FLa2. The 1601 bp FLa1 probe was amplified with primers ade2-P-UP-fw and ade2-P-UP-rv and the 1567 bp FLa2 probe was amplified using primers ade2-P-DW-fw and ade2-P-DW-rv B: Southern blot using a probe targeting lip. The 876 bp probe was amplified using the primers lipase-P-fw and lipase-P-rv. Marker: Bstll digested lambda DNA. FLa1: Upstream ade2 flank, FLa2: Downstream ade2 flank, lip: T. lanuginosus lipase gene, T: TtrpC terminator.

Additional file 4: Table S1. Additional media supplements. The supplements added to MM plates according to specific genotypes.

Additional file 5: Table S2. Oligonucleotides used for production of PCR fragments used for vector construction.

Additional file 6: Table S3. Plasmids constructed in this study.

Additional file 7: Figure S4. Construction of pMJ-005. 1. A 2311 bp $T$. reesei tku70 gene fragment was amplified with the primers tku70-fw and tku70-rv. 2. The fragment was introduced into TOPO ${ }^{\circledast}$ vector pCR2.1 (invitrogen) by following the manufacturers TOPO ${ }^{\circledR}$ cloning protocol, resulting in pMJ-001. 3. The pMJ-001 construct was linearized by digestion with Stul and SnaBI, digesting after the 1154th and 1171th bp of the tku70 coding sequence (CDS), respectively. 4. A 2762 bp amplicon of the Aspergillus nidulans acetamidase gene (amdS) was obtained by PCR with primers amdS-fw and amdS-rv and used as selective marker. The two primers each carried a 15 bp $5^{\prime}$-end sequence complimentary to one end of the linear pMJ-001 vector. 5. The PCR product was cloned into pMJ-001 by In-Fusion $\AA$ cloning (Clontech) by following the manufacturer's protocol, resulting in pMJ-005. The construct contains the amdS selective marker flanked by tku70 fragments of 1.3 and $1.0 \mathrm{~kb}$ in the pCR2.1 topo vector backbone.

Additional file 8: Table S4. Oligonucleotides used for diagnostic PCR, and Southern blot probes.

\section{Competing interests}

The authors declare that they have no competing interests.

\section{Authors' contributions}

MSJ has conducted all the experiments regarding this manuscript and has analyzed all data. The initial manuscript draft was composed by MSJ. DAS, PFJ and UHM have provided input for setup of the experimental design and have helped finalizing the manuscript. All authors read and approved the final manuscript.

\section{Acknowledgements}

This study was supported by grant 09-064967 from the Danish Council for Independent Research, Technology, and Production Sciences to UHM. MSJ's Ph.D project was funded by Novozymes A/S, the Department of Systems Biology (DTU) and the Danish research council via the Research program for Biotechnology (FOBI), Denmark.

\section{Author details}

${ }^{1}$ Department of Systems Biology, Center for Microbial Biotechnology, Technical University of Denmark, Building 223, DK-2800 Lyngby, Denmark.

${ }^{2}$ Novozymes A/S, Kroghoejsvej 36, DK-2880 Bagsvaerd, Denmark.

Received: 4 September 2013 Accepted: 10 February 2014 Published: 6 March 2014

\section{References}

1. Schuster A, Schmoll M: Biology and biotechnology of Trichoderma. Appl Microbiol Biotechnol 2010, 87:787-799.

2. Kubicek CP, Mikus M, Schuster A, Schmoll M, Seiboth B: Metabolic engineering strategies for the improvement of cellulase production by Hypocrea jecorina. Biotechnol Biofuels 2009, 2:19. 
3. Martinez D, Berka RM, Henrissat B, Saloheimo M, Arvas M, Baker SE, Chapman J, Chertkov O, Coutinho PM, Cullen D, Danchin EG, Grigoriev IV, Harris P, Jackson M, Kubicek CP, Han CS, Ho I, Larrondo LF, de Leon AL, Magnuson JK, Merino S, Misra M, Nelson B, Putnam N, Robbertse B, Salamov AA, Schmoll M, Terry A, Thayer N, Westerholm-Parvinen A, et al: Genome sequencing and analysis of the biomass-degrading fungus Trichoderma reesei (syn. Hypocrea jecorina). Nat Biotechnol 2008, 26:553-560.

4. Saloheimo M, Pakula TM: The cargo and the transport system: secreted proteins and protein secretion in Trichoderma reesei (Hypocrea jecorina). Microbiology 2012, 158(Pt 1):46-57.

5. Peterson R, Nevalainen H: Trichoderma reesei RUT-C30--thirty years of strain improvement. Microbiology 2012, 158:58-68.

6. Guangtao Z, Hartl L, Schuster A, Polak S, Schmoll M, Wang T, Seidl V, Seiboth B: Gene targeting in a nonhomologous end joining deficient Hypocrea jecorina. J Biotechnol 2009, 139:146-151.

7. Schuster A, Bruno KS, Collett JR, Baker SE, Seiboth B, Kubicek CP, Schmoll M: $A$ versatile toolkit for high throughput functional genomics with Trichoderma reesei. Biotechnol Biofuels 2012, 5:1.

8. Hartl L, Seiboth B: Sequential gene deletions in Hypocrea jecorina using a single blaster cassette. Curr Genet 2005, 48:204-211.

9. Dave MN, Chattoo BB: A counter-selectable marker for genetic transformation of the yeast Schwanniomyces alluvius. Appl Microbiol Biotechnol 1997, 48:204-207.

10. Bitan-Banin G, Ortenberg R, Mevarech M: Development of a gene knockout system for the halophilic archaeon Haloferax volcanii by use of the pyrE gene. J Bacteriol 2003, 185:772-778.

11. Millerioux Y, Clastre M, Simkin AJ, Marais E, Sibirny AA, Noel T, Creche J, Giglioli-Guivarc'h N, Papon N: Development of a URA5 integrative cassette for gene disruption in the Candida guilliermondii ATCC 6260 strain. J Microbiol Methods 2011, 84:355-358.

12. Santoso D, Thornburg R: Uridine 5'-Monophosphate Synthase Is Transcriptionally Regulated by Pyrimidine Levels in Nicotiana plumbaginifolia. Plant Physiol 1998, 116:815-821

13. Beck $P$, Dingermann T, Winckler T: Transfer RNA gene-targeted retrotransposition of Dictyostelium TRE5-A into a chromosomal UMP synthase gene trap. J Mol Biol 2002, 318:273-285.

14. Choquer M, Robin G, Le Pecheur P, Giraud C, Levis C, Viaud M: Ku70 or Ku80 deficiencies in the fungus Botrytis cinerea facilitate targeting of genes that are hard to knock out in a wild-type context. FEMS Microbiol Lett 2008, 289:225-232.

15. De Boer P, Bastiaans J, Touw H, Kerkman R, Bronkhof J, van den Berg M, Offringa R: Highly efficient gene targeting in Penicillium chrysogenum using the bi-partite approach in deltalig4 or deltaku70 mutants. Fungal Genet Biol 2010, 47:839-846.

16. Li ZH, Du CM, Zhong YH, Wang TH: Development of a highly efficient gene targeting system allowing rapid genetic manipulations in Penicillium decumbens. Appl Microbiol Biotechnol 2010, 87:1065-1076.

17. Atanasova L, Knox BP, Kubicek CP, Druzhinina IS, Baker SE: The polyketide synthase gene Pks4 of Trichoderma Reesei provides pigmentation and stress resistance. Eurkaryot Cell 2013, 12:1499-1508.

18. Fisher CR: Enzymology of the pigmented adenine-requiring mutants of Saccharomyces and Schizosaccharomyces. Biochem Biophys Res Commun 1969, 34:306-310.

19. Jin FJ, Maruyama J, Juvvadi PR, Arioka M, Kitamoto K: Adenine auxotrophic mutants of Aspergillus oryzae: development of a novel transformation system with triple auxotrophic hosts. Biosci Biotechnol Biochem 2004, 68:656-662

20. Du M, Battles MB, Nett JH: A color-based stable multi-copy integrant selection system for Pichia pastoris using the attenuated ADE1 and ADE2 genes as auxotrophic markers. Bioeng Bugs 2012, 3:32-37.

21. Silver JM, Eaton NR: Functional blocks of the ad-1 and ad-2 mutants of Saccharomyces cerevisiae. Biochem Biophys Res Commun 1969, 34:301-305.

22. Nielsen JB, Nielsen ML, Mortensen UH: Transient disruption of non-homologous end-joining facilitates targeted genome manipulations in the filamentous fungus Aspergillus nidulans. Fungal Genet Biol 2008, 45:165-170.

23. Hansen BG, Salomonsen B, Nielsen MT, Nielsen JB, Hansen NB, Nielsen KF, Regueira TB, Nielsen J, Patil KR, Mortensen UH: Versatile enzyme expression and characterization system for Aspergillus nidulans, with the Penicillium brevicompactum polyketide synthase gene from the mycophenolic acid gene cluster as a test case. Appl Environ Microbiol 2011, 77:3044-3051.

24. Geu-Flores F, Nour-Eldin HH, Nielsen MT, Halkier BA: USER fusion: a rapid and efficient method for simultaneous fusion and cloning of multiple PCR products. Nucleic Acids Res 2007, 35:e55

25. Fink GR, Hicks JB: Methods in Yeast genetics. Cold Spring Harb Lab Cold Spring Harb NY 1986, 565:578.

26. Gruber F, Visser J, Kubicek CP, de Graaff LH: The development of a heterologous transformation system for the cellulolytic fungus Trichoderma reesei based on a pyrG-negative mutant strain. Curr Genet 1990, 18:71-76.

27. Norholm MH: A mutant Pfu DNA polymerase designed for advanced uracil-excision DNA engineering. BMC Biotechnol [computer file] 2010, 10:21.

28. White $B$, Woodward D: A simple method for making disposable race tubes. Fungal Genet News/ 1995, 42:79.

doi:10.1186/1475-2859-13-33

Cite this article as: Jørgensen et al:: A novel platform for heterologous gene expression in Trichoderma reesei (Teleomorph Hypocrea jecorina). Microbial Cell Factories 2014 13:33.

\section{Submit your next manuscript to BioMed Central and take full advantage of:}

- Convenient online submission

- Thorough peer review

- No space constraints or color figure charges

- Immediate publication on acceptance

- Inclusion in PubMed, CAS, Scopus and Google Scholar

- Research which is freely available for redistribution 